\title{
Virtual Worlds as Platforms for Digital Entrepreneurship: The Role of Internal Governance and the Rule of Law
}

\author{
Anuragini Shirish ${ }^{1}$, Shalini Chandra ${ }^{2}$, and Shirish C. Srivastava ${ }^{3}$ \\ ${ }^{1}$ CERDI (Center for Studies and Research in Law of the Intangible), \\ Faculté Jean Monnet, Université Paris-Sud 11 \\ anuragini . tandalam@u-psud. fr \\ ${ }^{2}$ S P Jain School of Global Management, Singapore \\ shalini.chandra@spjain.org \\ ${ }^{3}$ Department of Operations Management and Information Technology, HEC, Paris \\ srivastava@hec.fr
}

\begin{abstract}
Based on the principles of 'rule of law' this research-in-progress paper theorizes the key role of internal governance procedures within virtual worlds for promoting digital entrepreneurship. By fostering adequate trust, internal governance procedures within VWs, provide the requisite amount of certainty, transparency, predictability and legitimacy to the transactions carried out within the VWs. In summary, this research proposes a plausible framework for conceptualizing the adoption and diffusion of VWs as a platform for digital entrepreneurship.
\end{abstract}

Keywords: Virtual Worlds, Rule of Law, Trust, IT Adoption, IT Diffusion, Internal Governance, Digital Entrepreneurship.

\section{Research Agenda}

Virtual Worlds (VWs) are fast emerging as attractive platforms for creating and conducting innovative business. Unlike traditional Internet based tools (such as e-commerce), which serve only as a medium for transaction and collaboration, VWs provide a comprehensive environment which could be leveraged for creating novel digital products and services. Analogous to real-world entrepreneurs, the emerging breed of digital entrepreneurs is looking to leverage the VW platform for producing and selling digital products and services within the VW. However, the potential adoption and diffusion of VWs among digital entrepreneurs appears be relatively less due to several reasons. One of the prime concerns limiting its entrepreneurial use is the fact that internal governance within VWs is seldom transparent, clear and predictable. The providers of VW often purport to have absolute discretion on the exercise of their powers to refuse user's interests/rights both under contract and property law. The basic and important questions that concern any digital entrepreneur are thus left vague and unanswered, leading to lack of trust in wanting to adopt VWs for entrepreneurial activities. 
Grounding the current study in the 'theory of the rule of law', and 'literature on virtual trust' we propose that similar to 'real world', digital entrepreneurs require predictable, legitimate and transparent internal governance procedures within the VW. It is well established in the real world that for fostering economic activity it is necessary to have the governance of persons (real or fictitious), based on the basic principles of the rule of law. The rule of law generally requires that the governing agencies announce and follow pre-negotiated procedures so that the expectations of the governor and the governed are clear, predictable and transparent. It is also well established that in societies where the rule of law is strong, economic development is progressive (Tamanaha, 2004). Moreover, rule of law becomes significant as a business enabler when virtual commerce expands (Balkin, 2004). In this research-inprogress poster paper, we propose a framework for conceptualizing the adoption and diffusion of VWs as a platform for digital entrepreneurship. We also suggest a plausible methodology for testing the formulated hypotheses.

\section{References}

Tamanaha, B.Z.: On the rule of law: History, politics, theory, p. 119. Cambridge University Press (2004)

Balkin, J.M.: Virtual Liberty: Freedom to Design and Freedom to Play in Virtual Worlds. Virginia Law Review 90(8), 2043-2098 (2004) 